\title{
Scientific models as works
}

\author{
Anita Coleman
}

Email: asc@u.arizona.edu 


\section{Acknowledgments}

$\checkmark$ Funded by the Office of the Vice President for Research, University of Arizona, Tucson (2001)

$\checkmark$ RA: Gaurav Gupta (ECE)

$\checkmark$ College of Engineering (Civil \& Mining)

$\checkmark$ UA Library http://models. library.arizona.edu/ 


\section{Background}

$\checkmark$ Traditional Libraries

- Three fundamentals of the catalog: author, subject, title

- Functions: Identification, Location, Collocation

$\checkmark$ Digital Libraries

- Resource discovery

- Creating Metadata for all types of objects

- Anything can be a document - information package that can be described 


\section{Challenge}

$\checkmark$ Neither textual nor conceptual stability can be taken for granted (Wilson, 2002, p.11)

$\checkmark$ Support the information retrieval process that accompanies teaching and learning functions/tasks

- NASA (1988) identified prerequisites for integration of research and model use 


\section{Definition of models}

$\checkmark$ Models

- Approx. 15 definitions of the word in OED Online

- Denotative meaning

- Connotative meaning

- Scientific Models

- "A simplified or idealized description or conception of a particular system, situation, or process (often in mathematical terms: so mathematical model) that is put forward as a basis for calculations, predictions, or further investigation."

Source: OED Online. (2002) 


\section{Questions}

$\checkmark$ Are scientific models "works"?

$\checkmark$ What is a scientific model and how can it be represented and retrieved in the library catalog?

$\checkmark$ Information behaviors related to scientific models 


\section{Properties of models}

$\checkmark$ Reflect reality

$\checkmark$ Small representations of reality

$\checkmark$ Simple representations of process/phenomenon

$\checkmark$ Closed

$\checkmark$ Reality is mathematically modeled

$\checkmark$ Most important features 


\section{Study of scientific models}

$\checkmark$ Science and engineering disciplines

$\checkmark$ Works: Intellectual artifacts, Creative process

$\checkmark$ Domain: Physical Geography (Atmosphere, Soils, Water Quality)

$\checkmark$ Examined: documents about models on the WWW and content analysis of models in published literature 


\section{Scientific models as works}

$\checkmark$ "A work is the intellectual content of a bibliographic entity; any work has two properties: a) the propositions expressed, which form ideational content and b) expressions of those propositions which form semantic content."

- Source: Smiraglia, Richard P. (2001) 


\section{Physical components}

- Texts (articles, bibliographies, reviews, reports, documentation)

- Datasets (data)

- Software (code)

- Services (databases) 


\section{Conceptual components}

$\checkmark$ Subject + other

- Focus of research: object, process

- Type of model

- Mathematical functions

- Instrumentation

- Fundamental theory or law

- Replication, revision, modification 


\section{What is a scientific model?}

\section{Information resource (form \& format)}

$\checkmark 1$. Theory or Hypothesis (text file or graphic diagram/image)

$\checkmark 2$. Observations and Measurements (data sets in various formats)

$\checkmark$ 3. Computer Hardware (descriptions only)

$\checkmark$ 4. Computer Software (this includes actual software executable code, other software essential for running the model code itself)

$\checkmark 5$. Documentation (technical and user guide that accompanies the software)

6. Animations (images, video)

$\checkmark$ 7. Visualizations (images, video)

8. Reports, Reviews, Annotations, Experiments, Articles (text)

$\checkmark$ 9. Tools (applets, services)

$\checkmark$ 10. Mathematic (algorithmic, numeric notation)

r 11. FAQs, Mailing Lists/Listservs, Discussion Groups, Events (various) 


\section{Scientific Model -Facets}

Concept: is an idea, the traditional subject (for example, calculus of variations)

( Object: the object studied in the model

Discipline: the major discipline to which this model belongs (may be determined either through author affiliations or other means)

Phenomenon: the phenomenon being modeled

Process: the process being modeled

MathRepresentation: the mathematical functions, equations used

Software: the software needed to run the model

FundamentalLaw: the fundamental laws that the model is based upon

Type: the type of model based on its purpose

Variable: number, types, conditions, and variables in this model

Problem: the problem the model is analyzing stated often as a question

Theory: is there an existing theory or research group to which this model belongs 


\section{Relationships}

$\checkmark$ Bibliographic relationships

- Tillett's Taxonomy of Bibliographic Relationships include derivative, equivalence, descriptive, whole-part, accompanying, sequential, and shared characteristic relationships

$\checkmark$ Subject relationships

- Facet analysis of subjects 


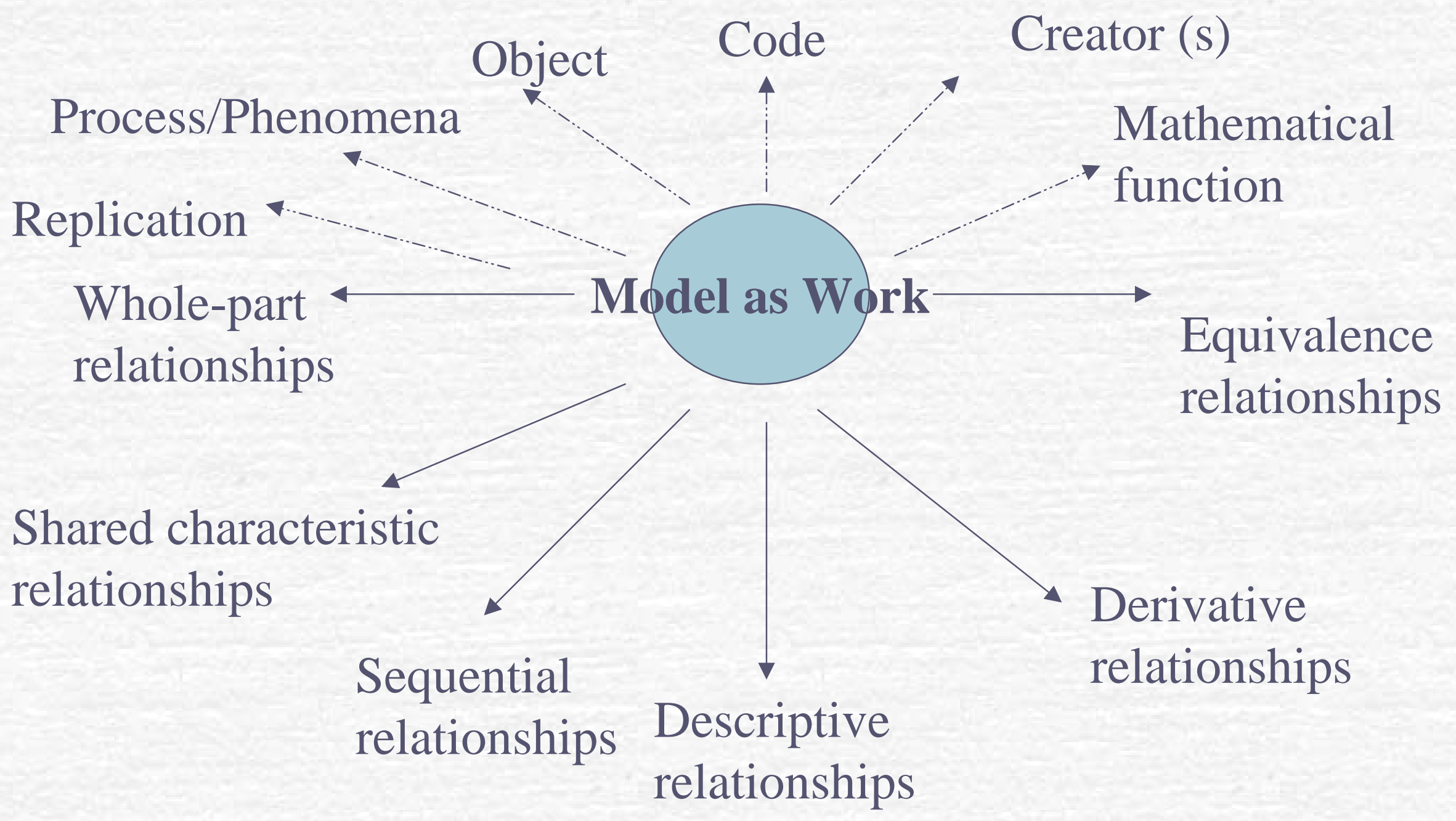




\section{Sample models - Water Quality}

$\checkmark$ Plug flow reactor models (Streeter \& Phelps, 1925)

$\checkmark$ BOD/DO (Streeter \& Phelps, 1925)

$\checkmark$ Oxygen-balance model (Streeter \& Phelps, 1925)

$\checkmark$ QUAL models (current version, Brown \& Barnwell, 1987)

r QUAL 11 


\section{Constitutive Models}

\begin{tabular}{|l|l|l|}
\hline $\begin{array}{l}\text { Search terms } \\
\text { \& strategy }\end{array}$ & $\begin{array}{l}\text { Sampling } \\
\text { Frame }\end{array}$ & $\begin{array}{l}\text { Number of hits } \\
\text { \& search date }\end{array}$ \\
\hline CM - Subject & OCLC & $9(10 / 01)$ \\
\hline CM - Google & WWW & 35,200 \\
\hline CR - Subject & OCLC & 2 \\
\hline CR - Keyword & OCLC & 33 \\
\hline Above & Sabio & 0 \\
\hline
\end{tabular}




\section{More questions}

$\checkmark$ What are shared characteristics of scientific models?

- How can they be used to sort and display results in library catalogs?

$\checkmark$ Which bibliographic relationships do representations about model replications best fit?

$\checkmark$ What's the difference between a work and a concept? (I dentifying boundaries) 


\section{References}

$\checkmark$ Coleman, A. (2002) Scientific models as works. C\&CQ 3/4: 129-159

r Smiraglia, R. (2001) The nature of a "work": I mplications for the organization of knowledge. Lanham: Scarecrow Press

$\checkmark$ Tillet, B. (1991) A taxonomy of bibliographic relationships. LRTS 35: 150-158 